\title{
Macroscopic Hematuria-A Leading Urological Problem in Patients on Anticoagulant Therapy: Is the Common Diagnostic Standard Still Advisable?
}

\author{
Artur A. Antoniewicz, Lukasz Zapała, Sławomir Poletajew, and Andrzej Borówka \\ Department of Urology, Medical Centre of Postgraduate Education, Miedzylesie Hospital, 04-749 Warsaw, Poland \\ Correspondence should be addressed to Sławomir Poletajew, slawomir.poletajew@gmail.com \\ Received 29 November 2011; Accepted 18 January 2012 \\ Academic Editors: A. M. El-Assmy and C. Magno
}

Copyright (C) 2012 Artur A. Antoniewicz et al. This is an open access article distributed under the Creative Commons Attribution License, which permits unrestricted use, distribution, and reproduction in any medium, provided the original work is properly cited.

\begin{abstract}
All urological standards of care are based on the past definition of the clinical importance of macroscopic hematuria. The aim of the study was to assess the phenomenon of iatrogenic hematuria in current clinical practice and analyze its origins in patients receiving anticoagulant drugs. Retrospective analysis of clinical documentation of 238 patients that were consulted for hematuria in $2007-$ 2009 by 5 consultant urologists was performed. In the group of 238 patients with hematuria, 155 (65\%) received anticoagulants. Abnormalities of urinary tract were found in 45 (19\%) patients. Estimated cost of a single neoplasm detection reached the value of 3252 Euro (mean 3-day hospitalization). The strong correlation between the presence of hematuria and anticoagulant treatment was observed. Authors suggest to redefine the present and future role of hematuria from a standard manifestation of serious urological disease to a common result of a long-term anticoagulant therapy.
\end{abstract}

\section{Introduction}

Hematuria, defined as a clearly visible change in urine color due to blood additives, may be a symptom of serious urinary tract disease. Thus, it should always be an urgent diagnostic matter for a clinician. The usage of anticoagulant or antiplatelet drugs is beneficial for patients with several diseases. However, serious complications may appear during such a therapy, including mucosal bleeding in the form of hematuria. Iatrogenic hematuria may be the reason for urological consultation and hospitalization in urological department, during which standard diagnostic procedures are usually performed.

All urological standards of care are based on the past definition of the clinical importance of presence of micro- and macroscopic hematuria. The growing number of iatrogenic hematuria reported in literature requires verification of guidelines for the management of patients with hematuria receiving any anticoagulant therapy. The aim of the study was to assess the phenomenon of iatrogenic hematuria in current clinical practice and analyze its origins in patients receiving anticoagulant drugs. The economic aspects of diagnosing hematuria were of special interest.

\section{Material and Methods}

Retrospective analysis of clinical documentation of $238 \mathrm{pa}-$ tients (132 men, 106 women) aged 18-99 years (average 57 years) was performed. All patients were consulted for hematuria in 2007-2009 by 5 consultant urologists in the following departments: Nephrology (43), Cardiology (27), General (23) and Vascular Surgery (11), Cardiosurgery (40), Hematology (34), Gynecology (11), and Internal Diseases (49). In the group of 238 patients with hematuria, there was a division on the subgroup of $155(65 \%)$ individuals, who received anticoagulant drugs-group A (Figure 1): oral anticoagulants (36\%), nonfractionized or fractionized heparins $(27 \%)$, and antiplatelet drugs: acetylsalicylic acid (21\%), clopidogrel (11\%), and ticlopidine (5\%). Another 83 (35\%) subgrouped patients, who presented with hematuria-group $\mathrm{B}$, did not receive any anticoagulant drugs. Hematuria was found predominantly in patients over 65 years $(67 \%)$, with 


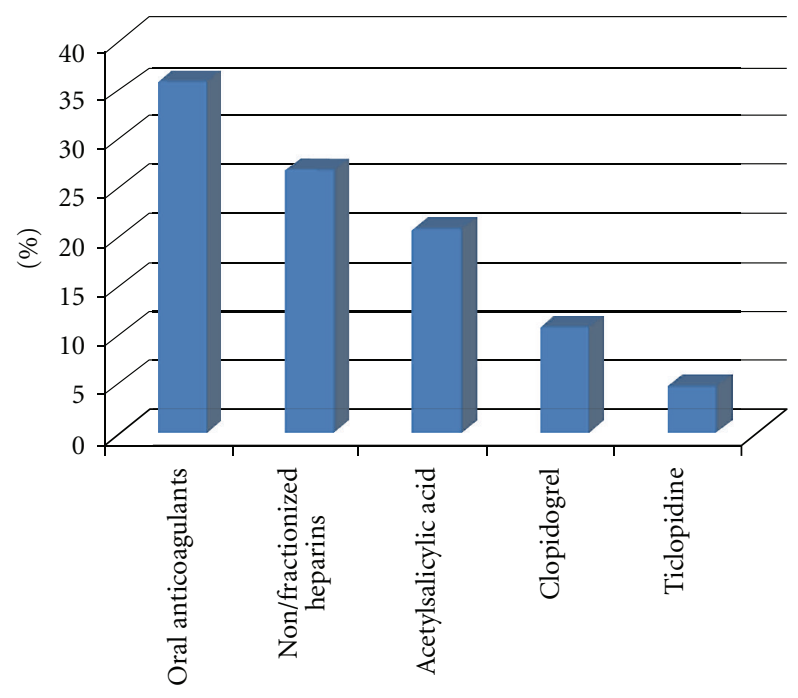

Anticoagulants

FIGURE 1: Individuals that received anticoagulant drugs—group A.

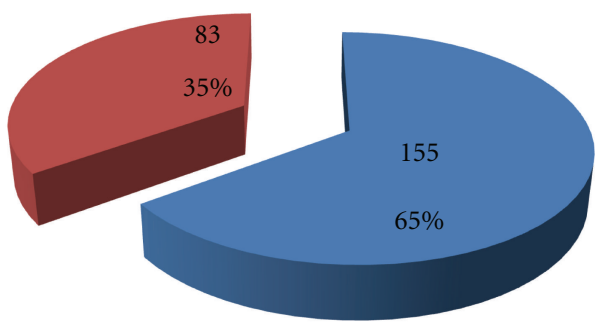

- Group A

- Group B

FIgURE 2: Hematuria as the top reason for urological consultation: the existence in group A versus group B.

concomitant diseases (71\%): hypertension, coronary heart disease, arrhythmia, and end-stage renal disease. The majority of patients had the full diagnostic panel performed ( $n=216,91 \%)$ including USG of urinary tracts, urography or contrast CT, and cystoscopy. The diagnostic results were analyzed with the special attention to the negative ones, in which no abnormalities in urinary tracts were found $(n=209,88 \%)$. Statistical analysis was performed using chisquare test with Yates correction.

\section{Results}

Hematuria was the top reason for urological consultation $(238 / 871,27 \%)$. It occurred predominantly in individuals on anticoagulant drugs representing group A (65\% versus $35 \%)$ (Figure 2). The diagnostic panel of greatest clinical importance was ultrasound examination and cystoscopy. Abnormalities of urinary tract were found in overall (group A

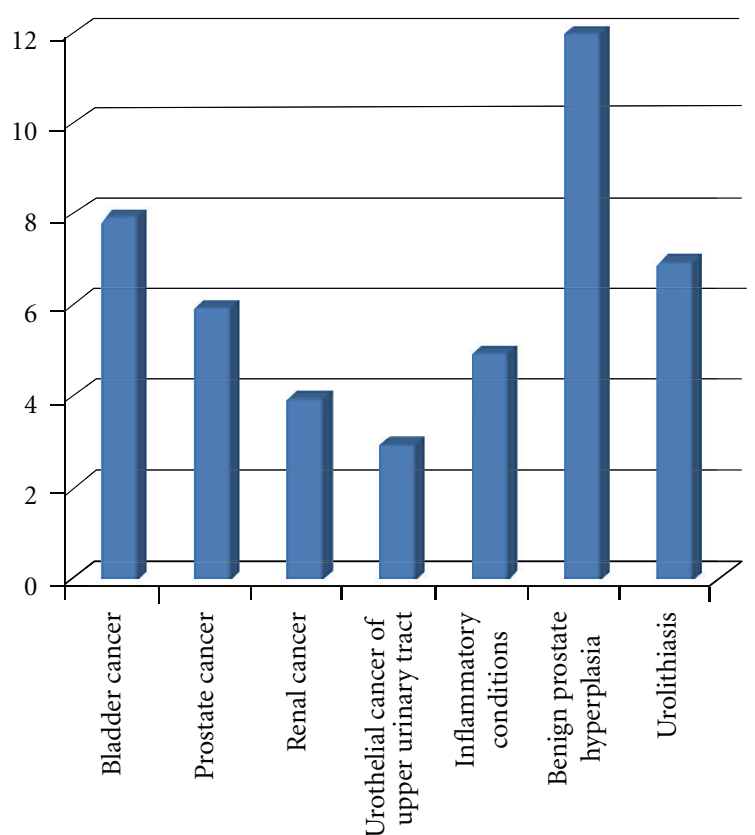

The abnormalities of urinary tracts

FIGURE 3: Abnormalities of urinary tract that were found in overall (group A plus group B).

plus group B) in 45 (19\%) patients (Figure 3): neoplasmsbladder cancer (8), prostate cancer (6), renal cancer (4), and urothelial cancer of upper urinary tract (3); inflammatory conditions (5); benign prostate hyperplasia (12); urolithiasis (7). The number of pathologies detected in the group A compared with group B was $8 \%$ and $16 \%$, respectively $(P=0.2)$. The common probability for $\mathrm{A}+\mathrm{B}$ groups of finding a tumor was low $(P 1=0.1$ in men, $P 2=0.06$ in women) with the highest one for diagnosing bladder cancer $(P 3=0.037$ in men, $P 4=0.02$ in women). The estimated cost of diagnostic procedures for hematuria per patient was 287 Euro, assuming average time of diagnostics as a 3-day hospitalization $(1 \mathrm{EUR}=4.5 \mathrm{PLN})$. The cost-effectiveness analysis (number of patients $\times$ cost of a single diagnostic panel/number of neoplasms detected) revealed that the cost of a single neoplasm detection reaches the value of 3252 Euro, which remains unacceptable for any health provider worldwide taking into consideration a predominant number of cases of iatrogenic hematuria (Table 1).

\section{Discussion}

Anticoagulants are increasingly used for the prevention and treatment of thromboembolic complications of vascular diseases [1]. Bleeding from the urinary tracts is naturally one of the most important complications of such a therapy.

Hematuria occurred to be the main reason for consultations in our material, being the matter of urological visits in various departments in $27 \%$ of cases. Hematuria is claimed 
TABle 1: Costs per patient according to National Health Fund.

\begin{tabular}{|c|c|c|c|c|}
\hline Payer & USG of urinary tracts & CT of abdominal cavity and pelvis & Cytoscopy & Urography \\
\hline National Heath Fund (NFZ) & 9,3 EUR & 66,67 EUR & 158,67 EUR & 52,4 EUR \\
\hline \multicolumn{4}{|c|}{ Overall (3-day hospitalization) } & 287 EUR \\
\hline
\end{tabular}

to involve $4 \%$ to $20 \%$ of all urological visits [2]. Those findings differ slightly, but our patients made up a specific group of great anticoagulants uptake due to serious health conditions (cardiology, cardiosurgery, and vascular surgery). Hematuria in a review of observational studies, average annual rates of fatal, and major and major/minor bleeding was 0,8 , 4,9 , and $15 \%$, respectively [3]. Gross hematuria occurred in $2,5 \%$ of the population [4]. However, the rate was higher in the older patients and when the indication for anticoagulant treatment was arterial disease [5]. About one-third of patients, who had bleeding complications, had more than one indication for anticoagulants, for example, peripheral and/or cerebral arterial disease, ischaemic heart disease, atrial fibrillation, and venous thromboembolic disease [5]. It is consistent with the results obtained in our study, in which in the majority of cases patients over 65 years were consulted, while the main reason for anticoagulants use was heart diseases. One should realize that especially in such a group the anticoagulant therapy needs to be administered not only carefully but also individually.

Bladder cancer was the most common oncological problem diagnosed in the presented study. Carcinoma, urolithiasis, benign prostatic hyperplasia (BPH), and inflammatory or infectious etiologies are most commonly identified [6-10]. Even though one should think of bladder cancer firstly in case of hematuria, the symptom itself is present predominantly in patients on anticoagulants. Lower tract bleeding occurs in $57 \%$ of cases, with the majority of these being gross hematuria, while upper tract bleeding occurs in $40 \%$ of the cases, with twice as much gross as microscopic hematuria [11]. Previously published studies demonstrated that gross and microscopic hematuria in late 1960s were documented in 4 to $24 \%$ and $40 \%$, respectively, of the patients on anticoagulant therapy with warfarin or heparin [12-14]. It was consistent with further studies from the 1990s, which revealed that gross hematuria occurred in $2 \%$ to $24 \%$ of patients receiving chronic anticoagulation with warfarin and/or aspirin for various indications $[11,15,16]$. Furthermore, it was aspirin that was in the majority of cases the reason of iatrogenic hematuria (78\% versus $62 \%$ in the warfarin group) [16]. Several randomized controlled trials, which enrolled 15406 patients receiving heparins, revealed that the complication of hematuria was present in 1,6\% of cases [17-23]. In the group treated with a high dose of LMW heparin, 5,8\% of patients developed hematuria, while in the group treated with a low dose of LMW heparin, 0,4\%. Furthermore, 4,7\% of patients receiving a high dose of LDU heparin presented with hematuria versus $0,2 \%$ of patients receiving a low dose of LDU heparin. The incidence of hematuria in patients treated with fibrinolytic agents approached 20 to $30 \%$ [24]. The overall percentage of individuals on anticoagulants in the consulted patients was higher in our material due to the several reasons. First of all, the patients were enrolled in our study from the departments, in which admitted patients are in great need of such a therapy. Furthermore, the studies published so far are often based on the material from general population lacking urological point of view focused on the possibility of a tumor presence in urinary tracts.

Urologists are often asked to evaluate the need of diagnostics in patients with hematuria, who are on anticoagulant therapy. The commonly accepted diagnostic standard is based on clinical assessment, ultrasonography and/or excretory urography (IVP), and cystoscopy. During imaging and urological procedures, an etiology is found in 17 to $82 \%$ of cases [6-10]. In our study, only $19 \%$ of consultations proved to be of urological matter, and that fact supports strongly the opinion according to which the probability of finding a tumor in the group of patients on anticoagulants is very low. Approximately $2 \%$ to $5 \%$ of patients with microscopic hematuria and $10 \%$ of patients with gross hematuria have urothelial carcinoma $[25,26]$. In a study of 1340 healthy men screened for hematuria, there was no correlation between the quantity of hematuria and urological disease severity [27]. Furthermore, hematuria produced by cancer and other serious diseases was frequently intermittent and appeared in small number of cases. In addition to that, the statistics change drastically along with enlarging the group of patients enrolled in the study and focusing on the conditions that individuals were treated from. In the large study of 1930 patients, nondiagnostic hematuria was found in the following: microscopic - $68 \%$ and macroscopic hematuria$52 \%$, respectively [28]. As mentioned above, these are arterial diseases, which due to the great need of anticoagulant therapy for patients suffering from those conditions and high prevalence of them in the population, coexist with iatrogenic hematuria [29]. Last and foremost is the fact that statistics in urological studies focus mainly on individuals referred by other clinicians to urological departments due to the highly possible urological origins of the patient's symptoms. In our study, we had a chance to examine the problem closely, due to the fact that the patients, who were consulted, suffered from different primary diseases.

Whether the prognosis with anticoagulants-associated hematuria is improved due to earlier detection of asymptomatic genitourinary lesions is uncertain [11]. Usually, the degree of hematuria is related to the degree of anticoagulation [11], although it may be the only manifestation of significant uropathological condition. Thus, some authors recommended a complete urological evaluation for all patients with nontraumatic anticoagulant-associated hematuria, having emphasized that a malignancy was found in $30 \%$ of the patients on anticoagulant therapy [11]. The statement that anticoagulants may serve as a potentiative enhancement for the detection of urological disease [11] seems to be quite risky 
nowadays, however. Moreover, as it was stated above, in our study, abnormalities of urinary tracts were found less frequently in patients receiving anticoagulants. The economic burden of investigating hematuria provokes a less intensive algorithm without loss of diagnostic efficacy [28]. However, some authors claimed that ultrasound in combination with IVP was recommended for maximal diagnostic efficacy [28]. In addition to that, cystoscopy shoud not be omitted on the basis of type of hematuria, age, or sex [28]. The American Urological Association recommended cystoscopy for all adults over 40 years old with microscopic hematuria and for those younger than 40 years with risk factors for developing bladder cancer [30]. However, such a policy leads to invasive procedures, for example, cystoscopy and imaging for $95 \%$ of patients with microscopic hematuria without malignancy detected [31]. The 3 types of hemorrhage that may occur in and around the urinary tract are retroperitoneal, intraluminal, and intrarenal [32]. Intraluminal bleeding often results in the formation of clots with possible painful passage or retention in the renal pelvis, which may simulate a neoplasm [33]. On the other hand, urothelial carcinoma of the bladder is frequently diagnosed in patients presenting with newonset hematuria. The authors are of the opinion that if the hematuria is rarely the revelator of a tumor in patients on anticoagulant therapy, a suggestion appears to limit the standard diagnostics procedures in those patients. The new standard would comprise ultrasound examination of urinary tracts and cystoscopy to rule out renal and bladder cancer, while common DRE would be in favor of excluding BPH and advanced prostate cancer. The main stress, however, should be put on the cost and effectiveness analysis that was performed in our study. The high costs of a single neoplasm detection in patients presenting with hematuria are caused by the great number of cases of iatrogenic hematuria that have to be excluded during diagnostic procedures. The alarming results make it clear that guidelines for anticoagulant treatment should be reconsidered.

The other element that requires consideration is the relevance of the extent of anticoagulation, which is influenced by the indication for treatment and the patient's compliance, dietary status, and concomitant drug therapy [16]. Some authors claimed that the incidence of bleeding episodes is directly correlated to the PT $[3,7,34]$. Moreover, the incidence of gross hematuria correlates with the degree of anticoagulation [16]. In those studies, it was again emphasized that despite the undeniable effect of anticoagulants on iatrogenic hematuria occurrence, each case of hematuria deserved a full attention of a urologist. Authors claim, however, that the only way to reduce the number of iatrogenic hematuria, which is diagnostic matter at urological wards, is to address that message to the clinicians of other fields, who prescribe anticoagulants for their patients.

\section{Conclusions}

In our study, the strong correlation between the presence of hematuria and anticoagulant treatment was observed. Urological origins of hematuria are more often present in patients not receiving anticoagulant drugs. Standard urological diagnostic procedure, as an expensive and invasive action, should be engaged only after critical analysis of influence of anticoagulant drugs on the presence of hematuria. Authors suggest to redefine the present and future role of hematuria from a standard manifestation of serious urological disease to a common result of a long-term or high-dose anticoagulant therapy. The controversy that remains is how to perform differential diagnosis between "tumor-induced" hematuria and "postmedication" hematuria. Further studies on this topic would be beneficial for clinicians and health care providers.

\section{References}

[1] J. Hirsch, "Drug therapy: oral anticoagulant drugs," The New England Journal of Medicine, vol. 324, pp. 1865-1875, 1991.

[2] A. J. Mariani, M. C. Mariani, C. Macchioni, U. K. Stams, A. Hariharan, and A. Moriera, "The significance of adult hematuria: 1,000 hematuria evaluations including a risk-benefit and cost-effectiveness analysis," Journal of Urology, vol. 141, no. 2, pp. 350-355, 1989.

[3] C. S. Landefeld and R. J. Beyth, "Anticoagulant-related bleeding: clinical epidemiology, prediction, and prevention," American Journal of Medicine, vol. 95, no. 3, pp. 315-328, 1993.

[4] C. D. Ritchie, E. A. Bevan, and J. Collier St. J., "Importance of occult haematuria found at screening," British Medical Journal, vol. 292, no. 6521, pp. 681-683, 1986.

[5] G. Palareti, N. Leali, S. Coccheri et al., "Bleeding complications of oral anticoagulant treatment: an inception-cohort, prospective collaborative study (ISCOAT)," The Lancet, vol. 348, no. 9025, pp. 423-428, 1996.

[6] J. T. Cuttino Jr, R. L. Clark, S. H. Feaster, and D. L. Zwicke, "The evaluation of gross hematuria in anticoagulated patients: efficacy of IV urography and cystoscopy," American Journal of Roentgenology, vol. 149, no. 3, pp. 527-528, 1987.

[7] S. J. Antolak Jr and G. T. Mellinger, "Urologic evaluation of hematuria occurring during anticoagulant therapy," Journal of Urology, vol. 101, no. 1, pp. 111-113, 1969.

[8] M. Barkin, W. Lopatin, S. Herschorn, and R. Comisarow, "Unexplained hematuria," Canadian Journal of Surgery, vol. 26, no. 6, pp. 501-503, 1983.

[9] U. Maier and M. Bergmann, "Hematuria under anticoagulant therapy as a symptom of urological diseases," Urologe A, vol. 19, no. 3, pp. 165-166, 1980.

[10] G. A. Schuster and G. A. Lewis, "Clinical significance of hematuria in patients on anticoagulant therapy," Journal of Urology, vol. 137, no. 5, pp. 923-925, 1987.

[11] J. G. van Savage, F. A. Fried, and E. Messing, "Anticoagulant associated hematuria: a prospective study," Journal of Urology, vol. 153, no. 5, pp. 1594-1596, 1995.

[12] D. H. Mosley, I. J. Schatz, G. M. Breneman, and J. W. Keyes, "Long-term anticoagulant therapy. Complications and control in a review of 978 cases," Journal of the American Medical Association, vol. 186, pp. 914-916, 1963.

[13] J. Roos and H. E. van Joost, "The cause of bleeding during anticoagulant treatment," Acta medica Scandinavica, vol. 178, no. 2, pp. 129-131, 1965.

[14] A. J. Zweifler, W. W. Coon, and P. W. Willis, "Bleeding during oral anticoagulant therapy," American Heart Journal, vol. 71, no. 1 , pp. 118-123, 1966. 
[15] J. C. Blankenship, "Bleeding complications of glycoprotein IIb-IIIa receptor inhibitors," American Heart Journal, vol. 138, no. 4, pp. S287-S296, 1999.

[16] Y. Avidor, A. Nadu, and H. Matzkin, "Clinical significance of gross hematuria and its evaluation in patients receiving anticoagulant and aspirin treatment," Urology, vol. 55, no. 1, pp. 2224, 2000.

[17] I. Lausen, R. Jensen, L. N. Jorgensen et al., "Incidence and prevention of deep venous thrombosis occurring late after general surgery: randomised controlled study of prolonged thromboprophylaxis," European Journal of Surgery, vol. 164, no. 9, pp. 657-663, 1998.

[18] M. T. Nurmohamed, R. Verhaeghe, S. Haas et al., "A comparative trial of a low molecular weight heparin (enoxaparin) versus standard heparin for the prophylaxis of postoperative deep vein thrombosis in general surgery," American Journal of Surgery, vol. 169, no. 6, pp. 567-571, 1995.

[19] K. Koppenhagen, J. Adolf, and M. Matthes, "Low molecular weight heparin in postoerative thrombosis prophylaxis," Wien Klin Wochenschr, vol. 82, pp. 349-353, 1994.

[20] R. S. McLeod, W. H. Geerts, K. W. Sniderman et al., "Subcutaneous heparin versus low-molecular-weight heparin as thromboprophylaxis in patients undergoing colorectal surgery: results of the Canadian colorectal DVT prophylaxis trial: a randomized, double-blind trial," Annals of Surgery, vol. 233, no. 3, pp. 438-444, 2001.

[21] J. P. Fricker, Y. Vergnes, R. Schach et al., "Low dose heparin versus low molecular weight heparin (Kabi 2165, Fragmin $($ ) ) in the prophylaxis of thromboembolic complications of abdominal oncological surgery," European Journal of Clinical Investigation, vol. 18, no. 6, pp. 561-567, 1988.

[22] S. Haas and C. W. Flosbach, "Prevention of postoperative thromboembolism with enoxaparin in general surgery: a German multicenter trial," Seminars in Thrombosis and Hemostasis, vol. 19, supplement 1, pp. 164-173, 1993.

[23] A. Encke and K. Breddin, "Comparison of a low molecular weight heparin and unfractionated heparin for the prevention of deep vein thrombosis in patients undergoing abdominal surgery," British Journal of Surgery, vol. 75, no. 11, pp. 10581063, 1988.

[24] J. A. Garcia-Vicente, J. Costa Pages, and P. Salva Lacombe, "Drug-induced hematuria," Medicina Clinica, vol. 100, no. 3, pp. 110-114, 1993.

[25] R. Bruyninckx, F. Buntinx, B. Aertgeerts, and V. Van Casteren, "The diagnostic value of macroscopic haematuria for the diagnosis of urological cancer in general practice," British Journal of General Practice, vol. 53, no. 486, pp. 31-35, 2003.

[26] R. A. Cohen and R. S. Brown, "Clinical practice. Microscopic hematuria," The New England Journal of Medicine, vol. 348, pp. 2330-2338, 2003.

[27] E. M. Messing, T. B. Young, V. B. Hunt et al., "Home screening for hematuria: results of a multi-clinic study," Journal of Urology, vol. 148, no. 2, pp. 289-292, 1992.

[28] M. H. Khadra, R. S. Pickard, M. Charlton, P. H. Powell, and D. E. Neal, "A prospective analysis of 1,930 patients with hematuria to evaluate current diagnostic practice," Journal of Urology, vol. 163, no. 2, pp. 524-527, 2000.

[29] P. L. Sanchez, M. H. Rubenstein, L. C. Harrell, P. J. ColonHernandez, J. K. Choo, and I. F. Palacios, "Frequency of gross hematuria shortly after percutaneous coronary intervention," American Journal of Cardiology, vol. 88, no. 1, pp. 71-73, 2001.

[30] G. D. Grossfeld, M. S. Litwin, J. S. Wolf et al., "Evaluation of asymptomatic microscopic hematuria in adults: the American Urological Association best practice policy-part II: patient ev- aluation, cytology, voided markers, imaging, cystoscopy, nephrology evaluation, and follow-up," Urology, vol. 57, no. 4, pp. 604-610, 2001.

[31] C. Tomson and T. Porter, "Asymptomatic microscopic or dipstick haematuria in adults: which investigations for which patients? A review of the evidence," British Journal of Urology International, vol. 90, no. 3, pp. 185-198, 2002.

[32] S. A. Kaufman and P. McLellan, "Urinary tract complications of anticoagulation therapy; 'pseudotumour' of the kidney," British Journal of Radiology, vol. 41, no. 483, pp. 180-185, 1968.

[33] R. L. Kroovand, T. E. Bell, and H. H. Kohler, "Coumadin induced hematuria simulating a renal pelvic tumor: case presentation and review of the literature," Journal of Urology, vol. 111, no. 2, pp. 223-224, 1974.

[34] W. W. Coon and P. W. Willis, "Hemorrhagic complications of anticoagulant therapy," Archives of Internal Medicine, vol. 133, no. 3, pp. 386-392, 1974. 


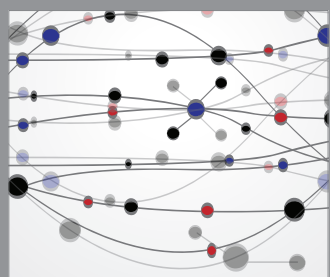

The Scientific World Journal
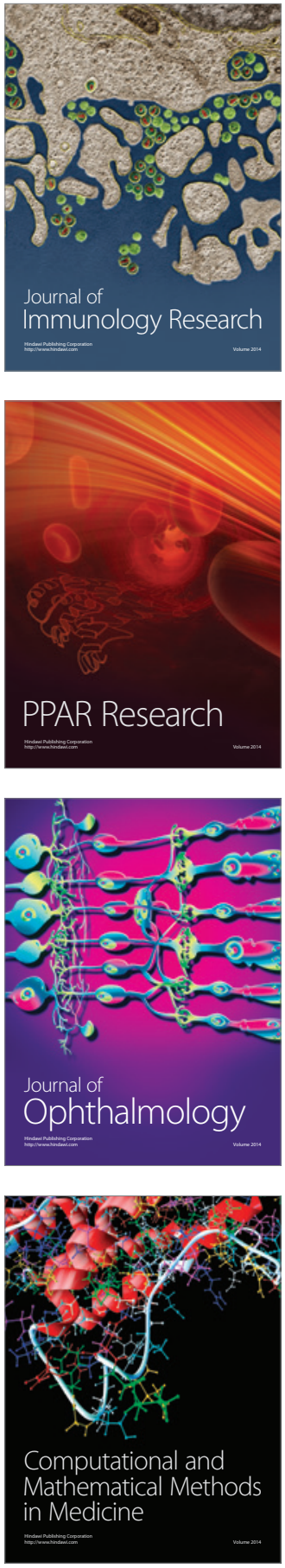

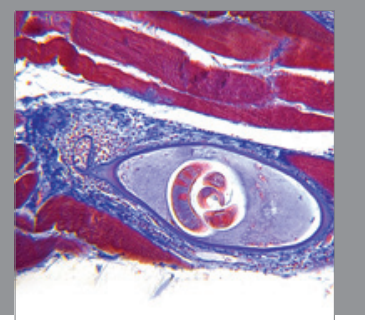

Gastroenterology

Research and Practice
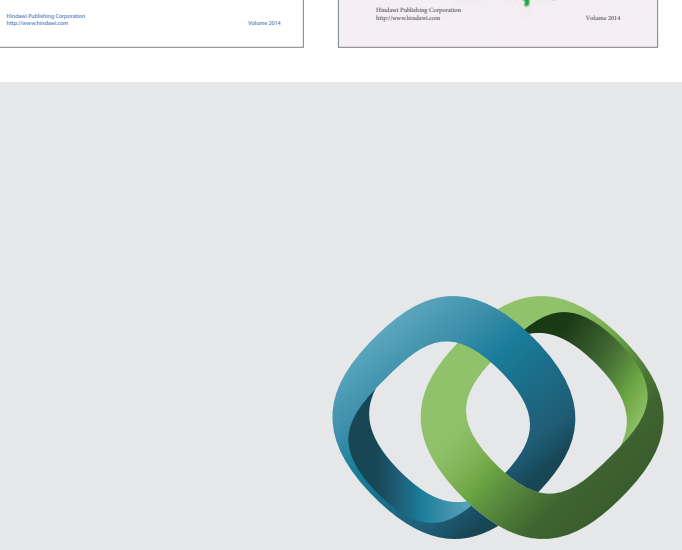

\section{Hindawi}

Submit your manuscripts at

http://www.hindawi.com
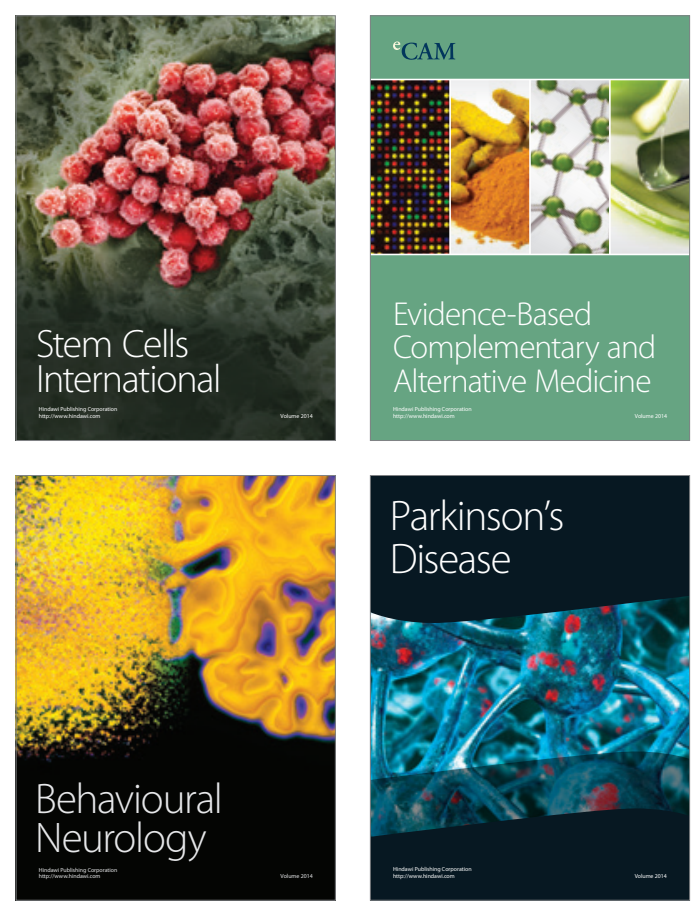

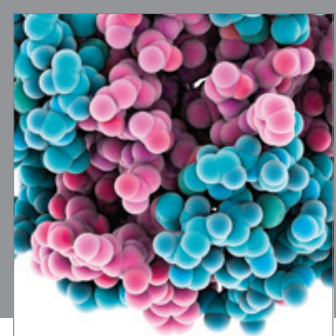

Journal of
Diabetes Research

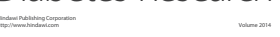

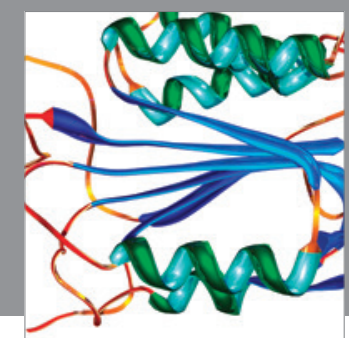

Disease Markers
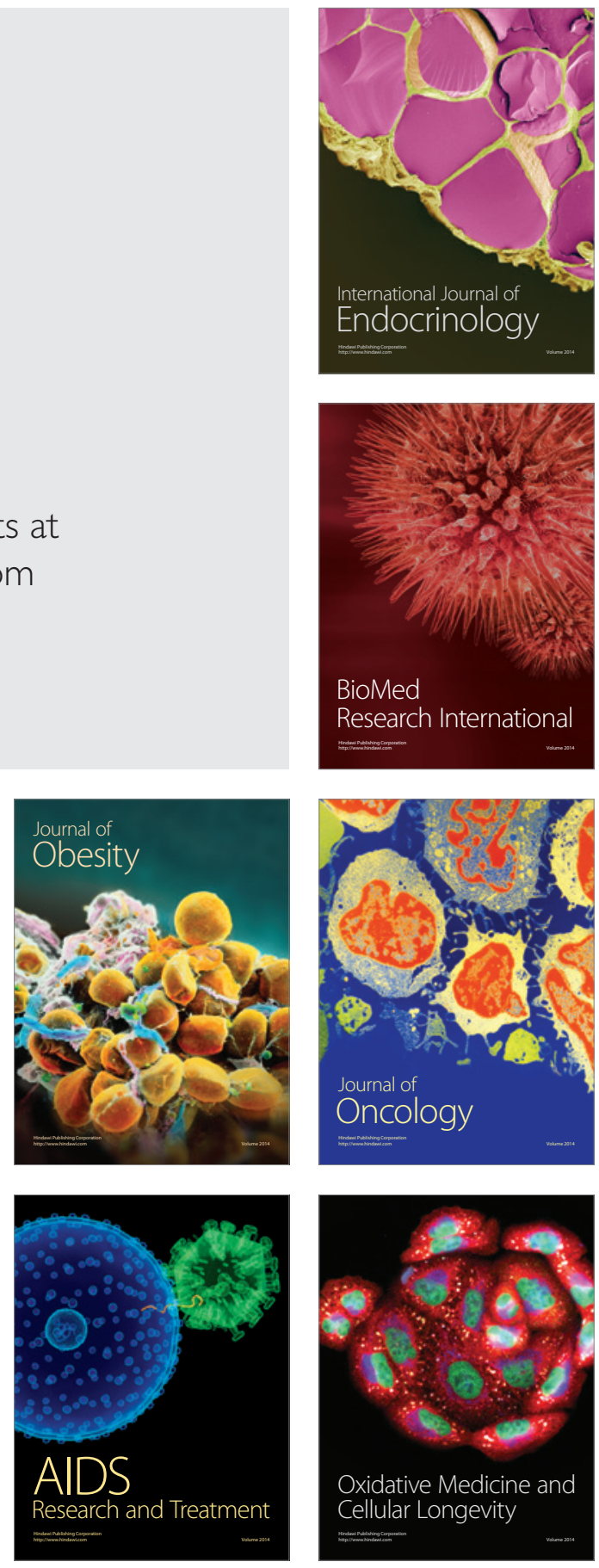